\title{
Synthesis and photophysical properties of fluorescent polystyrene containing 1,8-naphthalimide via atom transfer polymerization
}

\author{
Jin Zhengneng ${ }^{1}$, Lu Jianmei $^{1,2^{*}}$, Xu Qingfeng ${ }^{1}$, Wang Lihua ${ }^{1}$, Xia Xuewei $^{1}$ \\ ${ }^{1}$ Key laboratory of Organic Synthesis of Jiangsu Province, College of Chemistry and \\ Chemical Engineering, Suzhou University, Suzhou 215123, China, FAX: +86-0512- \\ 65882875, E-mail: lujm@suda.edu.cn. \\ 2Jiangsu Polytechnic University, Changzhou 213016, China
}

(Received: 18 October, 2006; published: 15 January, 2007)

\begin{abstract}
Fluorescent polystyrene was synthesized by Atom transfer polymerization with a novel 1,8-naphthalimide derivative as initiator. The optical characteristics of initiator and obtained polystyrene in DMF solution are discussed. Results indicate that both initiator and polystyrene can emit intensive green luminescence at $520 \mathrm{~nm}$, but the polymer has a higher quantum yield and energy yield.
\end{abstract}

\section{Introduction}

Recently, much attention have been paid to 1,8-naphthalimide derivatives because of their wide applications in structure coloration [1], light emitting diodes [2,3], laser media [4] and probes [5]. Compared to small molecules, polymeric 1,8-naphthalimide derivates are more promising for they have better optical stability, chromaticity and processability. Presently, there are three approaches to prepare such fluorescent polymers: (1) synthesis from different fluorescent monomers containing 1,8naphthalimide chromophore, then copolymerization with styrene, acrylonitrile or methyl methacrylate [6], Vladimir Bojinov and coworkers have done much in this aspect, they studied their fluorescent properties and chromaticity [7-9]; (2) use of Suzuki coupling or Yamamoto polycondensation reaction to get some conjugated polymers modified by 1,8-naphthalimide fluorophore, Lixiang Wang and coworkers have gotten some singe polymers for white electroluminescence diode by this method [10-12]; (3)Utilize chemical modification of commercially available polymers containing reactive groups, Deyue Yan and coworkers synthesized a new functional fluorescent polymer through the reaction of Poly(styrene-co-maleic anhydride) and 4amino-N-(2,4-dimethyl-phenyl)-1,8 -naphthalimide, the polymer has good thermal and optical property [13]. However, all the three methods listed above are uncontrolled, which may have some effects on their performance or limit further modification. So, it is worthwhile to synthesis such polymeric 1,8-naphthalimide derivatives via living polymerization.

As a relatively new living radical polymerization, Atom transfer radical polymerization (ATRP) has caught much interest in the synthesis of polymers with well-defined structure, lowly polydispersity and controlled molecular weight [14-16]. Especially, by using functional initiator, block, comb, graft, star, hyperbranched and surfaceanchored polymers can be easily prepared. If these initiators have optical and electronic properties, the obtained polymers may also have such properties. For example, Xiulin Zhu and coworkers used a number of azo-inititors capable of 
initiating styrene to obtain a new kind of polymers containing azo groups [17]. In the same way, series of initiators containing pyrene [18], anthracene [19] and $\mathrm{C}_{60}$ groups [20] were also applied to synthesis of polymers with special fluorescent properties. But as a material possessing strong luminance, 1,8-naphthalimides have never been reported in ATRP systems. Herein, we first used a novel kind of 1,8-naphthalimide derivative 2-(2,3-dihydro-2-(4-methoxy-phenyl)-1,3-dioxo-1H-phenalen-7-ylamino) ethyl-2-chloroacetate (DPDPECl) (the chemical structure is shown in Scheme 1) as initiator for atom transfer polymerization of styrene and obtained a greenish fluorescent polystyrene.

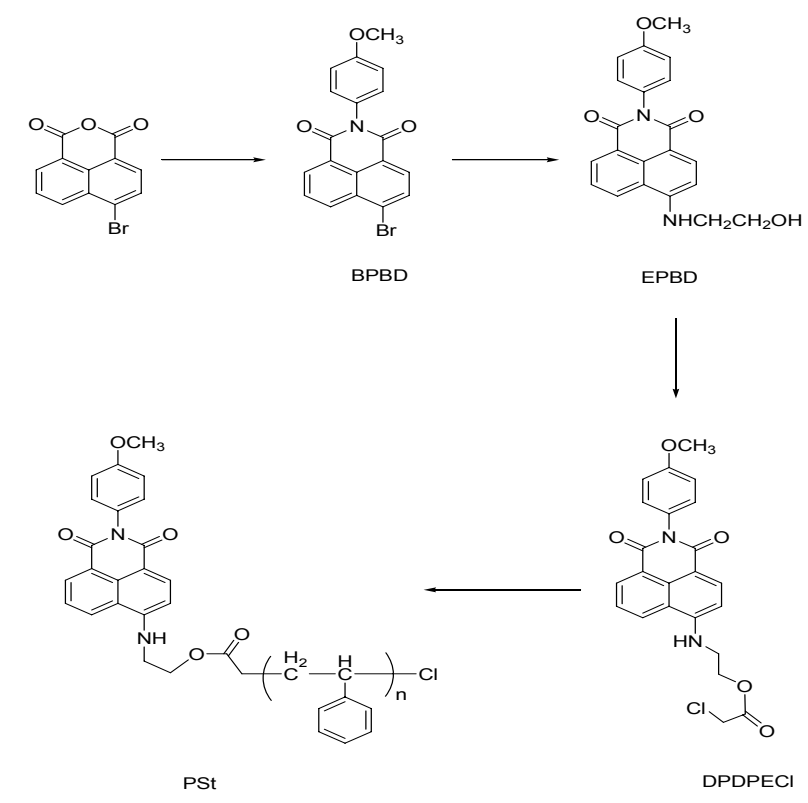

Scheme 1. The synthetic route for fluorescent polystyrene.

\section{Results and discussion}

\section{Synthesis of initiator DPDPECI}

The synthetic route of DPDPECI was outlined in Scheme 1. 6-bromo-2-(4-methoxyphenyl)-benzo[de]isoquinoline-1,3-dione (BPBD) was prepared by condensation of 4bromo-1,8-naphthalic anhydride with 4-meth- oxy-benzenamine in ethanol solution. 6-ethanolamino-2-(4-methoxy-phenyl)-benzo[de]isoquinoline-1,3-dione (EPBD) was obtained by nuclecophilic substitution of the $\mathrm{Br}$ group with ethanolamine in methoxyl ethanol solution. DPDPECI was obtained by the esterification of EPBD with acryloyl chloride.

In every reaction, TLC analysis was used to testify the purity. Compared to BPBD and EPBD, DPDPECl cannot be obtained by simple recrystallization from chlorobenzene, ethanol or ethyl acetate. Thus the pure DPDPECI was prepared by passing through a silica gel using $\mathrm{CHCl}_{3} / \mathrm{EtOAC}$ as eluent. Figure 1 shows the ${ }^{1} \mathrm{H}$ NMR spectrum of DPDPECl in $\mathrm{CDCl}_{3}$, the singlets at $3.86 \mathrm{ppm}$ originate from the protons of methoxy at position 1, the singlets at $3.76 \mathrm{ppm}$ and $4.16 \mathrm{ppm}$ are assigned to the protons of alkyl in the position 3 and 4, other singlets can also be well related to the rest of protons of DPDPECI. 


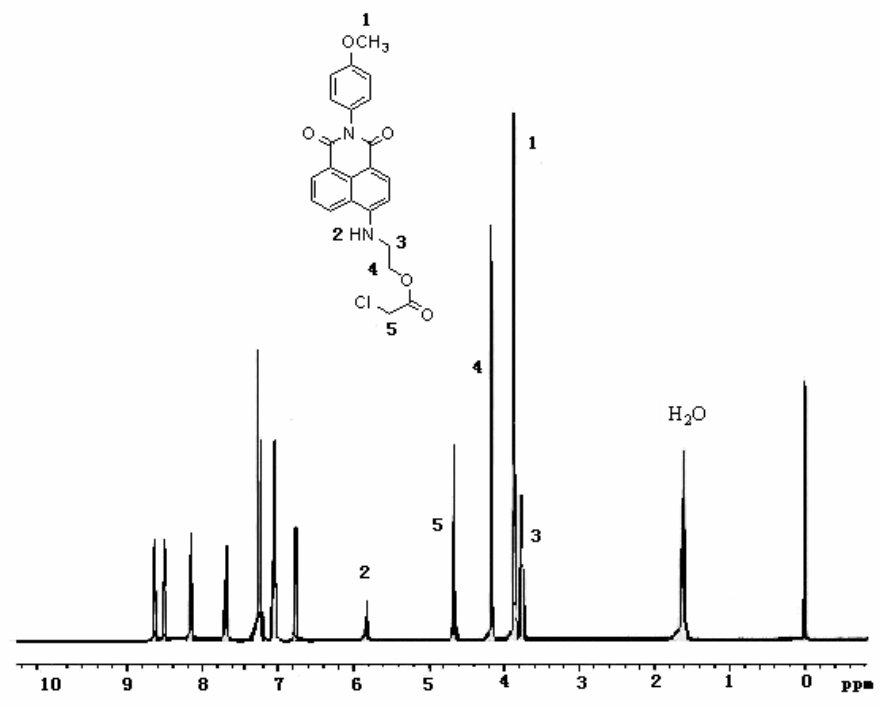

Fig. 1. ${ }^{1} \mathrm{H}-\mathrm{NMR}$ of DPDPECl in $\mathrm{CDCl}_{3}$.

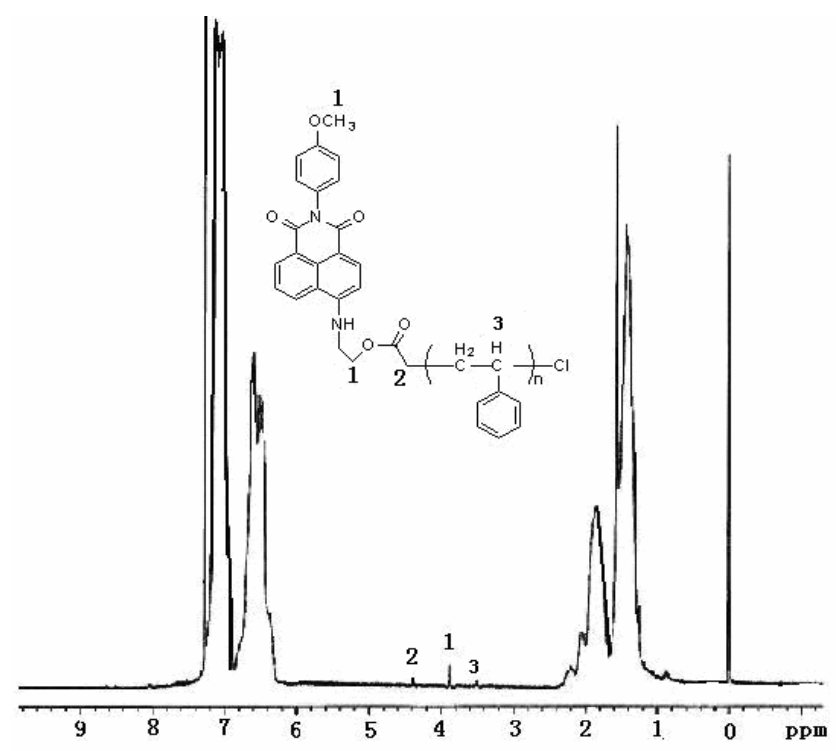

Fig. 2. ${ }^{1} \mathrm{H}-\mathrm{NMR}$ of $\mathrm{PSt}\left(\mathrm{M}_{\mathrm{n}}=18614, \mathrm{PDI}=1.421\right)$ in $\mathrm{CDCl}_{3}$.

Atom transfer radical polymerization of styrene

The polymerization was carried out using DPDPECI/CuCI/PMDETA as initiating system, styrene as monomer and cyclohexanone as solvent. The well-known mechanism of ATRP can be characterized by ${ }^{1} \mathrm{H}-\mathrm{NMR}$ spectrum, kinetic plots, polydispersity index and block extension copolymerization.

As per the character of ATRP, DPDPECl should be incorporated at one end of the polymer chain, while the other end remains a terminal halide. It can be testified by ${ }^{1} \mathrm{H}-\mathrm{NMR}$ spectrum. In Figure 2, signals at $3.90 \mathrm{ppm}$ are attributed to the protons in position 1 , including the protons of methoxy, the protons in the ester ethyl, signals at $4.4 \mathrm{ppm}$ are assigned to the protons in position 2, the protons of alkyl next to the ester carbonyl, signals at $3.5 \mathrm{ppm}$ are attributed to the terminal protons next to the halogen atoms. 


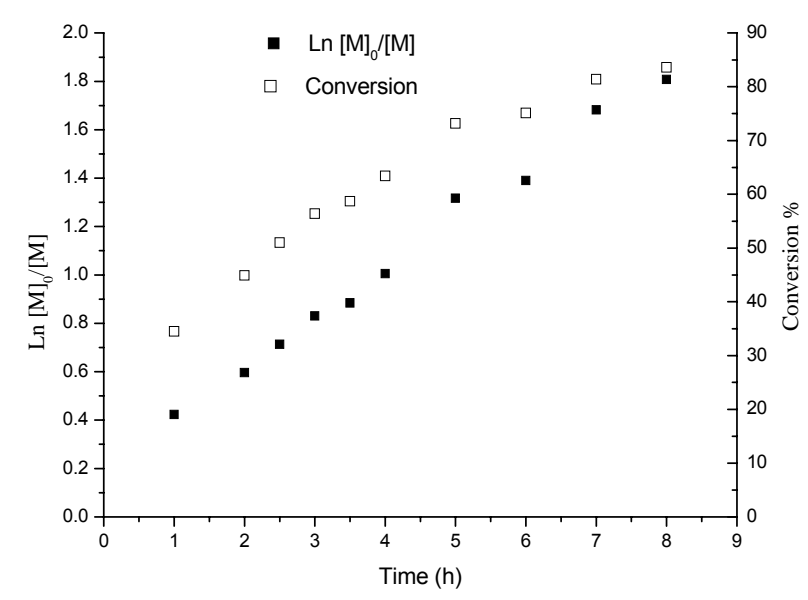

Fig. 3. Kinetic plot for the polymerization of styrene in cyclohexanone solution $(50 \% \mathrm{v} / \mathrm{v})$ at $100^{\circ} \mathrm{C}([$ styrene]/[DPDPECl]/ [CuCl]/[PMDETA]=200:1:1:1).

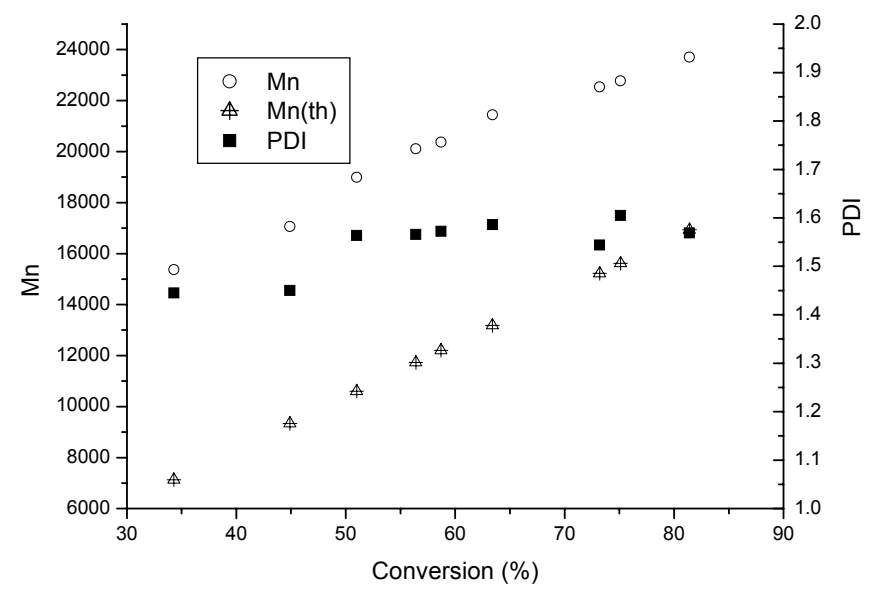

Fig. 4. Dependence of Mn and PDI with conversion for the polymerization of styrene initiated by DPDPECI. The condition was given in the caption of Figure 3.

The kinetic plot of polymerization at $100{ }^{\circ} \mathrm{C}$ is presented in Figure 3. It shows a typical linear variation of conversion and $\ln [\mathrm{M}]_{0} /[\mathrm{M}]$. ([M] $]_{0}$ and $[\mathrm{M}]$ denote the monomer concentrations at time 0 and $t$ with time. Such a behaviour indicates that there is a constant concentration of active species in the polymerization and first-order kinetics with respect to monomer. Figure 4 illustrates the dependences of number molecular weight and polydispersity index on monomer conversion. The $M_{n} s$ increase linearly with the conversion and the PDIs are in the region of 1.43 to 1.65 . In addition, it should be noted that the $M_{n} s$ measured by GPC are deviated from the theoretical values $\mathrm{Mn}_{(\mathrm{th})}\left(\mathrm{M}_{\mathrm{n}(\mathrm{th})}=[\mathrm{St}] /[\mathrm{DPDPECl}] \times\right.$ Conversion×104), which indicates a relatively slow initiation with respect to the fast propagation of monomer [21-23]. All of these results suggest a controlled polymerization.

To verify the living character of polymerization initiated by DPDPECl further, the resulting polystyrene was used for chain extension. The polymerization was carried 
out in solution (cyclohexanone) at $80^{\circ} \mathrm{C}$, with MMA as the second monomer and $[\mathrm{MMA}] /[\mathrm{PSt}] /[\mathrm{CuCl}] /[\mathrm{PMDETA}]=200: 1: 1: 1$. After 3 hours, the molecular weight measured by GPC increased from 15300 to 21200 and the PDI changed from 1.4 to 1.6 with the conversion up to $50 \%$. The GPC traces (Figure 5) show that the polymerization of the second monomer MMA for chain extension was successful.

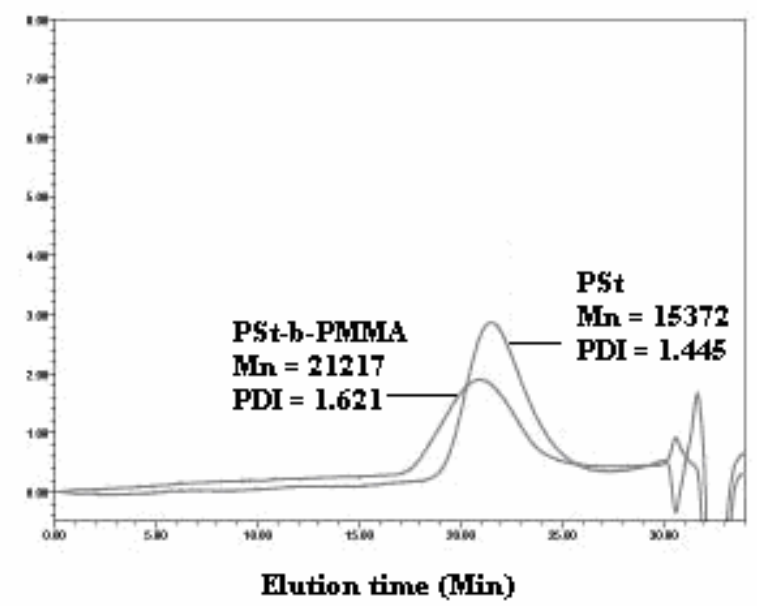

Fig. 5. GPC traces of PSt and relative PSt-b-PMMA copolymer.

\section{Spectral characteristics of PSt}

Due to the electron donor-acceptor interaction between the substituents at C-4 position and the carbonyl group from the imide structure, 1,8-naphthalimide derivatives have good fluorescent properties. For the polystyrene initiated by DPDPECl, every polymer chain contains one 1,8-naphthalimide group, and should have similar luminescence as the initiator DPDPECl. Table 1 presents the photophysical characteristics of DPDPECl and polystyrene in DMF solution.

Tab. 1. Photophysical Characteristics of DPDPECI and PSt.

\begin{tabular}{ccccccc}
\hline Substance & $\lambda_{A}, \mathrm{~nm}$ & $\log \varepsilon$ & $\lambda_{F}, \mathrm{~nm}$ & $\nu_{A}-\nu_{F}, \mathrm{~cm}^{-1}$ & $\varphi^{\mathrm{a}}$ & $\mathrm{E}_{\mathrm{F}}$ \\
\hline DPDPECl & 420 & 4.04 & 520 & 4578 & 0.61 & 0.49 \\
PSt $^{\mathrm{b}}$ & 430 & 3.47 & 520 & 4025 & 0.71 & 0.59
\end{tabular}

${ }^{\mathrm{a}}$ Relative to Fluorescein [24]

b The PSt refers to the polymer with $M_{n}=18600$ and PDI $=1.4$

Both DPDPECI and PSt in DMF solvent displayed a yellow-green colour and showed intensive green fluorescence under the radiation of UV light. The absorption maxima $\left(\lambda_{A}\right)$ were in the visible region, $420 \mathrm{~nm}$ and $430 \mathrm{~nm}$, respectively. The small distinction was probably caused by the chemical environment of chromospheres in DPDPECI and PSt. The fluorescent emission maxima for the initiator and polymer were the same $\left(\lambda_{F}=520 \mathrm{~nm}\right)$.

For 1,8-naphthalimide derivatives, Stokes shift $\left(v_{A}-v_{F}\right)$ is an important parameter, which indicates the difference in properties and structure of the dyes between the ground state $S_{0}$ and the first excited state $S_{1}$. The Stokes shift is calculated by Equation (1): 
$\left(v_{A}-v_{F}\right)=\left(1 / \lambda_{A}-1 / \lambda_{F}\right) \times 10^{7}$

Value for DPDPECI is larger than PSt, illustrates that more energy loss occurs during the transition from $S_{0}$ to $S_{1}$ in the small molecules.

Fluorescent quantum yield $(\phi)$ and energy yield of fluorescence $\left(E_{F}\right)$ show the ability of fluorescent materials to emit the absorbed light energy. $\Phi$ was determined using Fluorescein as a standard; $E_{F}$ is calculated by Equation (2):

$E_{F}=\phi_{F} \frac{\lambda_{A}}{\lambda_{F}}$

For PSt, the values of $\phi$ and $\mathrm{E}_{\mathrm{F}}$ are 0.71 and 0.59 , higher than those of DPDPECl. Such phenomenon was caused by the macromolecular structure of PSt, which hinder the conformational changes in the chromophoric system. This result is in agreement with the value change of Stokes shift; all this means less energy loss and stronger emitting strength for PSt [9].

\section{Conclusions}

In this paper, we have prepared a novel fluorescent polystyrene containing 1,8naphthalimide chromophore with atom transfer polymerization of styrene using DPDPECl/CuCl/PMDETA as initiating system and cyclohexanone as solvent. The initiator DPDPECl and polystyrene can emit intensive green fluorescence, but the polymer has higher quantum yield and energy yield.

\section{Experimental}

\section{Materials}

4-bromo-1,8-naphthalic anhydride (Liaoning Liangang Dyes Chemical Co. Ltd), 4methoxy-benzenamine (Sinopharm Chemical Reagent Co. Ltd), ethanolamine (Sinopharm Chemical Reagent Co. Ltd), Copper (II) sulfate pentahydrate $\left(\mathrm{CuSO}_{4} \cdot 5 \mathrm{H}_{2} \mathrm{O}\right.$ ) (Shanghai Zhenxing Chemical Reagent Factory), methoxyl ethanol (Sinopharm Chemical Reagent Co. Ltd ) and acryloyl chloride (Sinopharm Chemical Reagent Co. Ltd ) were used as received. Styrene (CP, Shanghai Chemical Reagent Co. Ltd) was purified by extracting with $5 \%$ sodium hydroxide aqueous solution, followed by washing with deionized water and drying with magnesium sulphate anhydrous overnight, and finally distillated in vacuum. Copper (I) chloride $(\mathrm{CuCl})$ (Shanghai Zhenxing Chemical Reagent Factory) was dissolved in hydrochloric acid, precipitated into a large amount of deionized water, filtered, washed with ethanol absolute, dried in vacuum. PMDETA (98\%, Jiangsu Liyang Jiangdian Chemical Factory) was dried with $4-A^{\circ}$ molecular sieve and distillated in vacuum. Cyclohexanone (AR) was dried with magnesium sulphate overnight and distilled in vacuum. Triethylamine (Sinopharm Chemical Reagent Co. Ltd) was distilled in vacuum. Other reagents were used without any further purification.

\section{Synthesis of 6-bromo-2-(4-methoxy-phenyl)-benzo[de]isoquinoline-1,3-dione (BPBD)}

4-bromo-1,8-naphthalic anhydride $2.5 \mathrm{~g}(9 \mathrm{mmol})$ and 4-methoxybenzenamine $2.5 \mathrm{~g}$ $(25 \mathrm{mmol})$ were refluxed in $100 \mathrm{ml}$ ethanol with vigorous stirring for 6 hours. The suspension was filtered to give a brown solid; recrystallization from chlorobenzene gave pale gray powder $3.0 \mathrm{~g}(88 \%)$. 
Synthesis of 6-ethanolamino-2-(4-methoxy-phenyl)-benzo[de]isoquinoline-1,3 dione (EPBD)

A mixture of BPBD $(1.5 \mathrm{~g}, 3.9 \mathrm{mmol})$, ethanolamine $(2.0 \mathrm{ml})$ and $\mathrm{CuSO}_{4} \cdot 5 \mathrm{H}_{2} \mathrm{O}(0.5 \mathrm{~g})$ was stirred in $50 \mathrm{ml}$ methoxyl ethanol to give a brown solution; the solution was allowed to reflux for 4 hours before it was poured into water, and a orange solid precipitated. After filtration, this solid was dried and recrystallized from chlorobenzene to give $1.2 \mathrm{~g}$ of orange-red powder (87\%).

Synthesis of 2-(2,3-dihydro-2-(4-methoxyphenyl)-1,3-dioxo-1H-phenalen-7- ylamino) ethyl 2-chloroacetate (DPDPECI)

EPBD $1.0 \mathrm{~g}(2.8 \mathrm{mmol})$ was dissolved in 100ml THF, dehydrated triethylamine $0.5 \mathrm{~g}$ $(5 \mathrm{mmol})$ and acryloyl chloride $(0.3 \mathrm{~g})$ in $15 \mathrm{ml}$ THF were added subsequently. The mixture was stirred at room temperature for 15 hours, poured into $500 \mathrm{ml}$ water. Filtered and dried, then the yellow powder was passed through a silica column using $\mathrm{CHCl}_{3} /$ EtOAc as eluent. Finally, DPDPECl $0.3 \mathrm{~g}$ was obtained (25\%).

\section{General polymerization}

$\mathrm{CuCl}, \mathrm{PMDETA}$, cyclohexanone, DPDPECl, styrene, and a small magnetic stirrer were added to a pressure tube. The tube was sealed and cycled between vacuum and $\mathrm{N}_{2}$ four times. Then the tube was sealed under $\mathrm{N}_{2}$ and placed in $\mathrm{a}$ thermostatically controlled oil bath. Samples were taken periodically for conversion and molecular weight analysis. The samples were dissolved in THF and precipitated into a large amount of hot methanol with several drops of $\mathrm{HCl}$ aqueous solution, filtrated and dried under vacuum. This process was repeated three times and then the greenish powder was used for analysis.

\section{Characterizations}

${ }^{1} \mathrm{H}-\mathrm{NMR}$ spectra were measured by INOVA $400 \mathrm{MHz}$ NMR spectrometer, $\mathrm{CDCl}_{3}$ as solvent. Purity of initiator was analyzed by TLC. Conversions for monomer were determined by gravimetry. Molecular weights and the polydispersity relative to polystyrene were measured using Waters 1515 GPC with THF as a mobile phase and with column temperature of $30^{\circ} \mathrm{C}$. UV-vis spectra were recorded on a PerkinElmerl-17 UV-vis instrument. Room temperature emission and excitation spectra were carried out using Edinburgh-920 fluorescence spectra photometer.

\section{Acknowledgements}

This work is supported by National Nature Science Foundation of China (No. 20476066).

\section{References}

[1] Filipova, T.Z.; Grabchev, I.; Petkov, I. J. Polym. Sci. Part. A: Poly. Chem. 1997, 35, 1069.

[2] Gan, J.A.; Song, Q.L.; Hou, X.Y.; Chen, K.C.; Tian, H. J. Photochem. Photobio. A: Chem. 2004, 62, 399.

[3] Kolosov, D.; Adamovich, V.; Djurovich, P.; Thompson, M.E.; Adachi, C. J. Am. Chem. Soc. 2002,124, 9945.

[4] Su, J.H.; Tian, H.; Chen, K.C.; Dyes Pigments 1996, 31, 69. 
[5] Kawai, K.; Kawabata, K.; Tojo, S.; Majima, T. Bioorg. Med. Chem. Lett. 2002, 12, 2363.

[6] Du, F.S.; Cai, H.; Li, Z.C.; Li, F.M. J. Polym. Sci. Part. A: Poly. Chem. 1998, 36, 1111.

[7] Bojinov, V.; Grabchev, I.; Dyes Pigments 2003, 59, 277.

[8] Grabchev, I.; Bojinov, V.; Betcheva, R. J. Appl. Polym. Sci. 2001, 81, 2463.

[9] Grabchev, I.; Petkov, C.; Bojinov, V. Macromol. Mater. Eng. 2002, 287, 904.

[10] Liu, J.; Zhou, Q.G.; Cheng, Y.X.; Geng, Y.H.; Wang, L.X.; Ma, D.G.; Jing, X.B.; Wang, F.S. Adv. Mater. 2005, 17, 2974.

[11] Tu, G.L.; Zhou, Q.G.; Cheng, Y.X.; Wang, L.X.; Ma, D.G.; Jing, X.B.; Wang, F.S. Appl. Phys. Lett. 2005, 85, 2172.

[12] Tu, G.L.; Mei, C.Y.; Zhou, Q.G.; Cheng, Y.X.; Geng, H.H.; Wang, L.X.; Ma, D.G.; Jing, X.B.; Wang, F.S. Adv. Funct. Mater. 2006, 16, 101.

[13] Wang, K.C.; Huang, W.; Xia, P.; Gao, C.; Yan, D.Y. React. Funct. Polym. 2002, $52,143$.

[14] Coessens, V.; Pintauer, T.; Matyjaszewski, K. Prog. Polym. Sci. 2001, 26, 337.

[15] Matyjaszewski, K.; Xia, J.; Chem. Rev. 2001, 101, 2921.

[16] Kamigaito, M.; Ando, T.; Sawamoto, M.; Chem, Rev, 2001, 101, 3689.

[17] Wang, G.; Zhu, X.L.; Cheng, Z.P.; Zhu, J. J. Polym. Sci. Part. A: Polym. Chem. 2005, 43, 2358.

[18] Michael, R.K.; Michel, R.G. Chem. Commun. 2000, 1711.

[19] Zhang, H.Q.; Bert, K.; Rob, van der L. Macromolecules 2002, 35, 2261.

[20] Zhou, P.; Chen, G.Q.; Li, C.Z.; Du, F.S.; Li, Z.C.; Li, F.M. Chem. Commun. 2000, 797.

[21] Matyjaszewski, k.; Wang, J.L.; Grimaud, T.; Shipp, D.A. Macromolecules 1998, $31,1527$.

[22] Li, M.; Min, K.; Matyjaszewski, K. Macromolecules 2004, 37, 2106.

[23] Sumerlin, B.S.; Neugebauer, D.; Matyjaszewski, K. Macromolecules 2005, 38, 702.

[24] Demas, J.N., Crosby, G.A. J. Phys. Chem. 1971, 75, 991. 